\title{
Factors Associated With Sexual Dysfunction in Newly Married Women Referred to the Urban Health Centers of Zanjan, Iran: A Cross-Sectional Study
}

\author{
Leila Alimohammadi $^{(\mathbb{D}}$, Fatemeh Zarei $^{2}$, Mojgan Mirghafourvand $^{3^{*}(\mathbb{D}}$
}

\begin{abstract}
Objectives: Sexual dysfunction is highly prevalent nowadays and is a threat to healthy marital life. The aim of the present study was to determine the factors associated with sexual dysfunction in newly married women referred to the urban health comprehensive services centers of Zanjan, Iran in 2015-2016.

Materials and Methods: This descriptive-analytical cross-sectional study was conducted on 300 women married in the last 5 years who referred to the health centers in Zanjan. The participants were selected through cluster sampling method. Data were collected using a socio-demographic characteristics questionnaire and the Female Sexual Function Index. Descriptive and analytical statistics including independent $t$ test, one-way ANOVA, and the general linear model were used to analyze the data.

Results: Sexual dysfunction was found in $66 \%$ of the women. The frequency of sexual dysfunction was $33 \%$ for arousal, $31 \%$ for pain, $28.7 \%$ for desire, $23 \%$ for satisfaction, $3 \%$ for vaginal lubrication, and $16.3 \%$ for orgasm dimensions. According to the general linear model, breastfeeding and contraceptive method had a significant relationship with the mean score of sexual function.

Conclusions: About two-thirds of women in their early years of marriage had sexual dysfunction, and given its impact on all aspects of marital life, it seems necessary to include sexual counseling in the pre-marital counseling program.

Keywords: Sexual function, Prevalence, Newly married women
\end{abstract}

\section{Introduction}

Sexual function is a complex interaction of neurovascular and hormonal factors and is influenced by biological characteristics, interpersonal relationships as well as cultural, traditional, and social features (1). Sexual disorders refer to disruptions in psycho-social tendencies and changes in characteristics associated with sexual response cycle. It is considered as a cause for anxiety and problems in interpersonal relationships (2) that affects well-being, quality of life, and lifestyle of women (24). According to the Diagnostic and Statistical Manual of Mental Disorders, sexual dysfunction has been introduced as a side effect in stages which are known as sexual response cycle or intercourse-associated pain (5). This definition emphasizes that human sexual response cycle model is a chronological sequence and consists of several coordinated stages including sexual desire, arousal, orgasm, and satisfaction (6).

Epidemiology of sexual dysfunction in women is poorly understood because few population-based studies were carried out on the prevalence, determinants, and consequences of women's sexual dysfunction $(7,8)$. In addition, socio-cultural barriers, taboos and false beliefs make it difficult to estimate sexual dysfunction prevalence which seems actually underestimated (9). However, population-based studies regarding the prevalence of sexual dysfunction in women have been increased in the past decade in order to better identify and understand the impact of this problem on women's lives, and these studies have been conducted in different countries, cultures, and age groups (10). It is estimated that 40 to $50 \%$ of women in the United States are suffering from sexual dysfunction and the problems associated with sexual dissatisfaction which have been reported in $87 \%$ of older women $(7,8)$. Rouzi et al showed that the prevalence of sexual dysfunction among Saudi and non-Saudi female healthcare providers was high (11).

In Iran, few studies have been done on the prevalence of sexual dysfunction in women (12). Ramezani Tehrani et al showed that the prevalence of sexual dysfunction in women in four provinces of Qazvin, Kermanshah, Golestan, and Hormozgan was $27.3 \%$ and dysfunction in the stages of desire, arousal, lubrication, and orgasm was $35.6 \%, 39.9 \%, 18.9 \%$, and $27.3 \%$, respectively; and $15.2 \%$ of them were dissatisfied with their sex life (13). The prevalence of sexual disorders in other studies was reported to be between 31 and $66 \%(12,14,15)$.

Dissatisfaction with sexual performance has always been one of the few causes of divorce and marital conflict. Bolhari et al showed that sexual problems are the cause of 
divorce in $88 \%$ of the study population (16). A report of Islamic Consultative Assembly also shows an increasing rate of divorce despite the declining trend of marriage rate, and the ratio of divorce to marriage had a tremendous growth in the early 2000 s compared to early 1990 s, as $8.76 \%$ in late 1990 s reached $14.13 \%$ and $25.6 \%$ in Tehran in 2009. According to this report, sexual dysfunction and sexual dissatisfaction of couples are mentioned as the most important causes of divorce, highlighting an urgent need for correct and timely decisions, as well as counseling and life skills training before, during, and after marriage as an effective way to strengthen the foundation of families and reduce divorce $(17,18)$.

Given the importance of sexual function in continuation of marital life and the lack of similar research on newlywed women, the present study aimed to determine the prevalence of sexual dysfunction and its associated factors in the target group in the urban health comprehensive services centers of Zanjan to pave the way for future studies and training programs in order to further consolidate marital ties and family foundations.

\section{Materials and Methods}

Study Type and Participants

This cross-sectional study was conducted on newly married women who visited the urban health centers of Zanjan city in 2015-2016.

Inclusion criteria included the age of 20-35 years, marital life duration of 6 months to 5 years, and education of at least high school. Exclusion criteria were special physical and mental diseases of woman or her husband, taking medications that affect the sexual function (currently or in the past six months), addiction of one of the couple to narcotic drugs and psychotropic substances (according to the participants' expression), occurrence of adverse events during the past six months (death of a child or loved one, serious illness of a close relative, prison sentence of one family member, history of depression, etc), conditions which make impossible the continuation of couples living together (such as violence, divorce, and administrative missions), forced marriage, and emotional divorce (according to the participants' expression).

The sample size was estimated to be 198 people according to a study conducted by Mohammad-Alizadeh et al (12). Considering $P=0.66, \alpha=0.05, d=0.1$, and the design effect of 1.5 , the final sample size was calculated to be 300 people.

\section{Sampling}

In this study, each of the health centers in the city $(n=38)$ was considered as a cluster from which 300 women were selected through the stratified random sampling method based on inclusion criteria and by taking into account the ratio of sample to the population. Therefore, the researcher attended the health centers and after coordination with the authorities, obtained the list of couples married over the past six months to 5 years, including their phone numbers, with the help of a health professional as a collaborator. Then, based on the number of samples determined for each center according to the population covered, 1.5 times the size of the quotas for each center were randomly selected using www.random.org website; the reason for which was to have the possibility of replacing if some women were not willing to participate in the study. Then the selected women were called by phone through which the researcher introduced herself and stated the general objective and a brief explanation of the research, and enrolled the women willing to participate in the study. In the next step, according to the scheduled time, the volunteers attended the health centers of Zanjan and received more information regarding the objectives, the importance of research, and the informed written consent form. The consent form was obtained and then the socio-demographic characteristics questionnaire and the Female Sexual Function Index were completed by the participants through self-administered method. Since the initial contact with the women was performed by the healthcare provider of the region, the rate of women's participation was high (about 70\%).

\section{Data Collection Tools}

In this study, the socio-demographic characteristics questionnaire and the Female Sexual Function Index (FSFI) were used for data collection through selfcompleted method.

The socio-demographic characteristics questionnaire was developed by research team and included questions about age of woman and her husband, length of marriage, contraceptive method, couple's education and job, living with spouse's family, previous marriage of the wife and husband, sufficiency of monthly income, satisfaction of husband job, breastfeeding, and pregnancy history.

The FSFI designed by Rosen et al was used to measure sexual function. Validity and reliability of this questionnaire were confirmed by Mohammadi et al in Iran and a Cronbach Alpha of $\geq 70$ has been reported (19). The FSFI is a standard scale for assessing sexual function in women during the past four weeks. This scale includes 19 items which measure 6 different aspects of sexual function including desire, arousal, vaginal lubrication, orgasm, satisfaction, and pain. The score of items 3-14 and 17-19 is $0-5$ and for items $1,2,15$, and 16 is $1-5$. The scores of each domain were calculated by summing the scores of items in each domain and then multiplying it by the coefficient of each domain (The coefficient value was 0.6 for desire domain, 0.3 for arousal and lubrication domains, and 0.4 for orgasm, satisfaction, and pain domains). The scores for the desire domain ranged from 2.1 to 6 and for other domains ranged from 0 to 6 . The total score obtained from summing all the 6 mentioned domains ranged from 2 to 36. Higher scores showed better sexual function. The score equal or less than 28 was considered as sexual dysfunction. 
Data Analysis

The obtained data were analyzed using SPSS version 21.0. Descriptive statistics including mean, standard deviation, frequency, and percentage were used to describe the sexual function and its sub-domains. The normality of quantitative data was assessed by skewness and kurtosis. The relationship between sociodemographic characteristics and the total score of sexual function was investigated using bivariate statistical tests including independent $t$ test and one-way ANOVA. Then, variables which had a significant relationship with sexual performance $(P<0.05)$ were entered in the general linear model. $P<0.05$ was considered significant.

\section{Results}

A total of 300 newlywed women living in Zanjan participated in the study. The mean (SD) age of the women and their husbands was 24.7 (6.8) and 24.5 (7.0), respectively. About half of women (45\%) and their spouses $(41 \%)$ had high levels of education. The shortest and the longest marriage length among participants were 1 year (8\%) and 5 years (32\%), respectively. About 85\% of the participants in this study were housewives. Most of their husbands were self-employed and half of them (54\%) were satisfied with their jobs, and based on participants report, about $69 \%$ had a sufficient monthly income. Most of the participants (95\%) and $93 \%$ of the husbands had experienced first marriage. About a third of women lived with the spouse's family. The most common method of contraception in women participated in the study was the natural method (24\%). $86 \%$ of the participants were nonpregnant and $74 \%$ had no breastfeeding infant.

In this study, one-way ANOVA was used for multimode variables and independent $t$ test for binary variables. Based on one-way ANOVA, there was no significant relationship between sexual function and women's education $(P=$ $0.638)$ and job $(P=0.242)$, husbands' education $(P=$ $0.539)$ and job $(P=0.900)$, length of marriage $(P=0.217)$, husbands' job satisfaction $(P=0.151)$, and efficiency of monthly income for living expenses $(P=0.705)$. In addition, according to independent $t$ test, no significant relationship existed between sexual function and previous marriage of the wife and husband $(P=0.677)$, pregnancy $(P=0.184)$, and living with spouse's family $(P=0.956))$. There was a statistically significant association between sexual function and breastfeeding $(P=0.02)$ according to independent $t$-test and between sexual function $(P=$ 0.035 ) and contraceptive method based on one-way ANOVA (Table 1).

The mean (SD) total score of sexual function in this study was 24.7 (6.6). The prevalence of sexual dysfunction in the participants was $66 \%$. The highest and the lowest frequency of dysfunction were observed in arousal (33.0\%) and orgasm (16.3\%) components, respectively. The frequency of dysfunction in other components of sexual function was $28.0 \%$ for desire, $18.3 \%$ for lubrication,
Table 1. Socio-demographic Characteristics and Their Relationship With Sexual Function in Participants $(n=300)$

\begin{tabular}{|c|c|c|c|}
\hline Variable & Number & Mean (SD) & $P$ \\
\hline Age $(y)$ & & & 0.232 \\
\hline 25 and lower & 120 & $24.2(6.5)$ & \\
\hline $25-30$ & 104 & $24.5(7.0)$ & \\
\hline 30 and higher & 76 & $25.8(6.1)$ & \\
\hline Education & & & 0.638 \\
\hline Under diploma & 46 & $24.4(6.6)$ & \\
\hline Diploma & 118 & $24 / 2(6.2)$ & \\
\hline More than diploma & 136 & $25.1(7.0)$ & \\
\hline Occupation & & & 0.242 \\
\hline Housewife & 254 & $24.5(6.7)$ & \\
\hline Employed & 46 & $25.7(6.2)$ & \\
\hline Income & & & 0.705 \\
\hline Favorable & 43 & $25.3(7.8)$ & \\
\hline Fairly favorable & 208 & $24.7(6.7)$ & \\
\hline Unfavorable & 49 & $24.1(5.2)$ & \\
\hline The first marriage of women & & & 0.198 \\
\hline Yes & 285 & $24.6(6.7)$ & \\
\hline No & 15 & $26.3(4.7)$ & \\
\hline Pregnant & & & 0.184 \\
\hline Yes & 41 & $23.5(6.2)$ & \\
\hline No & 259 & $24.9(6.7)$ & \\
\hline Contraception & & & 0.035 \\
\hline No contraception & 60 & $23.4(7.2)$ & \\
\hline Pill & 14 & $25.4(3.7)$ & \\
\hline Condom & 79 & $26.1(6.2)$ & \\
\hline IUD & 22 & $25.6(5.1)$ & \\
\hline Injection & 11 & $28.7(2.7)$ & \\
\hline Withdrawal & 113 & $23.9(7.0)$ & \\
\hline Breastfeeding & & & 0.002 \\
\hline Yes & 78 & $22.9(8.9)$ & \\
\hline No & 222 & $25.6(5.3)$ & \\
\hline Husband's age (y) & & & 0.985 \\
\hline Lower than 30 & 122 & $24.7(6.2)$ & \\
\hline $30-35$ & 133 & $24.7(6.8)$ & \\
\hline 35 and higher & 45 & $24.9(7.3)$ & \\
\hline Husband's education & & & 0.539 \\
\hline Under diploma & 76 & $24.6(5.7)$ & \\
\hline Diploma & 97 & $25.3(5.4)$ & \\
\hline More than diploma & 124 & $24.3(7.9)$ & \\
\hline Living with husband's family & & & 0.956 \\
\hline Yes & 75 & $24.7(6.6)$ & \\
\hline No & 225 & $24.7(6.6)$ & \\
\hline Husband's occupation & & & 0.900 \\
\hline Employee & 73 & $24.9(7.8)$ & \\
\hline Unemployed & 162 & $24.4(6.4)$ & \\
\hline Freelancer & 9 & $26.3(3.3)$ & \\
\hline
\end{tabular}




\begin{tabular}{|c|c|c|c|}
\hline Worker & 40 & $25.3(4.8)$ & \\
\hline Other & 16 & $24.4(8.0)$ & \\
\hline The first marriage of husband & & & 0.677 \\
\hline Yes & 280 & $24.7(6.7)$ & \\
\hline No & 20 & $25.1(4.4)$ & \\
\hline Duration of marriage & & & 0.217 \\
\hline 1 & 23 & $24.4(7.2)$ & \\
\hline 2 & 48 & $25.8(4.2)$ & \\
\hline 3 & 61 & $25.1(6.6)$ & \\
\hline 4 & 73 & $23.9(7.8)$ & \\
\hline 5 & 95 & $25.1(6.4)$ & \\
\hline Satisfaction from husband's job & & & 0.151 \\
\hline Yes & 161 & $25.3(6.8)$ & \\
\hline No & 75 & $23.5(5.7)$ & \\
\hline Moderate & 64 & $24.7(7.1)$ & \\
\hline
\end{tabular}

Abbreviations: SD, standard deviation

Table 2. The Status of Sexual Function and its Dimensions in Participants $(n=300)$

\begin{tabular}{lcc}
\hline Variable & Mean (SD) & $\begin{array}{c}\text { Disorder Prevalence } \\
\text { No. (\%) }\end{array}$ \\
\hline Total score of sexual function & $24.7(6.6)$ & $198(66.0)$ \\
Desire & $3.6(0.9)$ & $86(28.7)$ \\
Arousal & $3.7(1.2)$ & $99(33 / 0)$ \\
Lubrication & $4.0(1.3)$ & $55(18.3)$ \\
Orgasm & $4.3(1.5)$ & $49(16.3)$ \\
Satisfaction & $4.6(1.1)$ & $69(23.0)$ \\
Pain & $4.2(1.6)$ & $93(31.0)$
\end{tabular}

Abbreviations: SD, standard deviation

\section{0\% for satisfaction, and $31.0 \%$ for pain (Table 2).}

The significant variables based on one-way ANOVA and independent $t$-test were entered into the general linear model, according to which, contraceptive method and breastfeeding variables were associated with sexual function (Table 3).

\section{Discussion}

Sexual dysfunction in women of reproductive age has been investigated in numerous studies, but due to the impact of this problem on all aspects of marital life, this study aimed to determine the prevalence of sexual dysfunction and the related factors in newlywed women in Zanjan.

In this study, $66 \%$ of the participants had sexual dysfunction. The results of this study are consistent with those of the study by Mohammad-Alizadeh et al (prevalence of sexual dysfunction was 66\%) (12). Other studies have reported a prevalence of $31 \%$ to $69 \%$ for sexual dysfunction (13-15,20-23). Given the inadequacy and inappropriateness of education-based programs in the field of sexual problems, especially in pre-marriage
Table 3. The Relationship Between Socio-demographic Characteristics and Sexual Function in Participants According to the General Linear Model $(n=300)$

\begin{tabular}{lll}
\hline Variable & B (Cl 95\%) & P Value \\
\hline Contraception (Reference: withdrawal) & -- & -- \\
No contraception & $-0.9(-2.9$ to 1.0$)$ & 0.333 \\
Pill & $1.4(-2.1$ to 4.9$)$ & 0.432 \\
Condom & $2.2(0.3$ to 0.4$)$ & 0.019 \\
IUD & $1.4(-1.4$ to 4.3$)$ & 0.338 \\
Injection & $4.3(0.3$ to 8.3$)$ & 0.031 \\
Breastfeeding (reference: no) & -- & -- \\
Yes & $-3.5(5.2$ to -1.9$)$ & $<0.001$ \\
\hline
\end{tabular}

counseling, as well as the cultural background of this region, the relatively high prevalence of sexual dysfunction in newlywed women is not far-fetched.

In this study, the highest and lowest prevalence among different domains of sexual function were related to arousal (33\%) and orgasm (16\%), respectively. The results of the studies conducted by Mazinani et al (24), Ramezani Tehrani et al (13), Mohammad-Alizadeh et al (12), and Laumann et al (7) were in line with this study. In these studies, the most and the least disorder were also seen in the arousal and orgasm dimensions. In a study by Shokrollahi et al (25), arousal had the lowest prevalence. However, in the study of Safarinejad (14) and Yekeh Fallah and Goodarzi (26), the most prevalent was observed in the orgasm dimension.

It seems that the lack of sufficient knowledge and skill about sexual issues and fear of sexual relationship at the beginning of marriage can affect arousal and orgasm. On the other hand, in all countries and religions, sexual relationship is wrapped in an aura of superstitious beliefs and talking about it is associated with a sense of shame. Perhaps cultural, ethnic, religious, and racial differences in expression and dealing with sexuality in each country including Iran and even in each region are the reasons for the difference in statistics.

In this study, there was a significant relationship between the contraceptive method and sexual dysfunction, while no significant relationship existed in the study of Mazinani et al (24) and Hoseini Tabaghdehi et al (27). In the present study, the participants were young and at the beginning of marriage and hence mostly used natural contraceptive method. Given the commonness of natural contraceptive method and fear of pregnancy in the early years of marital life as well as inadequate skills for a satisfying sexual relationship among women in the study, experiencing disorder in each of the stages of sexual response cycle is not unexpected.

In this study, there was a significant relationship between breastfeeding and the prevalence of sexual dysfunction. The results of a study by Sehhatie et al (28) were consistent with this finding. Meanwhile, Pourakbar and Yazdi (29) 
emphasized in their study that children at home affect sexual dysfunction.

In the present study, no significant association was found between sexual dysfunction and other sociodemographic variables such as couples' age and job, length of marriage, couples' previous marriage, living with husband's family, pregnancy, husband's job satisfaction, income adequacy, and couples' education. According to Nooroozi et al (30), no significant relationship was found between age, education, length of marriage, job, and experience of previous marriage and sexual problems. However, a statistically significant relationship existed between age, education, length of marriage, job, and the experience of previous marriage and sexual function in the study of Yekeh Fallah and Goodarzi (26). Moreover, no significant relationship was found among the variables of education and length of marriage and sexual function in the studies of Mazinani et al (24), Karbasi-Afshar et al (31), and Bolourian and Ganjloo (20).

Since the present study was performed on women, it is recommended that similar research be carried out on the prevalence of sexual dysfunction in men population in Zanjan in the same group to complete information, so that the results can be used for planning, training and treatment of young couples in the form of therapeutic-educational approaches including pre-marriage counseling, sexual issues training, and family issues counseling with emphasis on culture. A good sexual function can increase the general health of families and the community and hence the foundation of families. Although this study was done on a community sample, it seems impossible to generalize the results to all women with the same condition, and it requires studies on a wider level. On the other hand, since the issue of sexuality is based on patterns and sociocultural beliefs of each region, studies with qualitative methodology can provide a better understanding in this regard.

\section{Conclusions}

The data obtained from this study showed that sexual dysfunction in newlywed women is a common and remarkable phenomenon, however, since the individuals had socio-cultural and familial constraints in addressing this issue, and in many cases, lack of information or incomplete information and lack of adequate skills in this field as well as irrational beliefs in this field are additional factors for the occurrence of marital conflicts, therefore, identification and diagnosis of these disorders and awareness of factors affecting this process can solve marital problems and help improve the couples' relations.

\section{Conflict of Interests}

Authors declare that they have no conflict of interests.

\section{Ethical Issues}

This study was approved by the Ethics Committee of
Tabriz University of Medical Sciences (No. TBZMED. REC.1394.764).

\section{Financial Support}

Tabriz University of Medical Sciences, Tabriz, Iran.

\section{Acknowledgements}

This article is a part of a student thesis, which was sponsored by Tabriz University of Medical Sciences. The researcher and his colleagues sincerely appreciate the vice chancellor for research of Tabriz University of Medical Sciences, head and personnel of healthcare centers of Zanjan. All women who participated in our research are also appreciated.

\section{References}

1. Fourcroy JL. Female sexual dysfunction: potential for pharmacotherapy. Drugs. 2003;63(14):1445-1457.

2. American Psychiatric Association. Diagnostic and statistical manual of mental disorders (DSM). Washington, DC: American Psychiatric Association; 1994:143-147.

3. Taylor MJ, Rudkin L, Bullemor-DayP, Lubin J, Chukwujekwu C, Hawton K. Strategies for managing sexual dysfunction induced by antidepressant medication. Cochrane Database Syst Rev. 2013(5):Cd003382. doi:10.1002/14651858. CD003382.pub3

4. Jha S, Thakar R. Female sexual dysfunction. Eur J Obstet Gynecol Reprod Biol. 2010;153(2):117-123. doi:10.1016/j. ejogrb.2010.06.010

5. First MB, Wakefield JC. Diagnostic criteria as dysfunction indicators: bridging the chasm between the definition of mental disorder and diagnostic criteria for specific disorders. Can J Psychiatry. 2013;58(12):663-669. doi:10.1177/070674371305801203

6. Basson R, Berman J, Burnett A, et al. Report of the international consensus development conference on female sexual dysfunction: definitions and classifications. J Urol. 2000;163(3):888-893.

7. Laumann EO, Paik A, Rosen RC. Sexual dysfunction in the United States: prevalence and predictors. JAMA. 1999;281(6):537-544.

8. Azadzoi KM, Siroky MB. Neurologic factors in female sexual function and dysfunction. Korean J Urol. 2010;51(7):443449. doi:10.4111/kju.2010.51.7.443

9. Anastasiadis AG, Davis AR, Ghafar MA, Burchardt M, Shabsigh R. The epidemiology and definition of female sexual disorders. World J Urol. 2002;20(2):74-78. doi:10.1007/s00345-002-0272-5

10. McCool ME, Theurich MA, Apfelbacher C. Prevalence and predictors of female sexual dysfunction: a protocol for a systematic review. Syst Rev. 2014;3:75. doi:10.1186/20464053-3-75

11. Rouzi AA, Sahly N, Sawan D, Kafy S, Alzaban F. The prevalence of sexual dysfunction in the female health care providers in Jeddah, Saudi Arabia. Sci Rep. 2015;5:7905. doi:10.1038/srep07905

12. Mohammad-Alizadeh-Charandabi S, Mirghafourvand M, Asghari-Jafarabadi M, Tavananezhad N, Karkhaneh M. Modeling of socio-demographic predictors of sexual function in women of reproductive age. Journal of Mazandaran University of Medical Sciences. 
2014;23(110):238-242.

13. Ramezani Tehrani F, Farahmand M, Simbar M, Malek Afzali H. Factors associated with sexual dysfunction; a population based study in Iranian reproductive age women. Arch Iran Med. 2014;17(10):679-684. doi:0141710/aim.008

14. Safarinejad MR. Female sexual dysfunction in a populationbased study in Iran: prevalence and associated risk factors. Int J Impot Res. 2006;18(4):382-395. doi:10.1038/ sj.ijir.3901440

15. Bahrami N, Alizadeh S, Bahrami S. Sexual dysfunctions and associated factors in women of reproductive age. Journal of Shahid Beheshti School of Nursing \& Midwifery. 2012;21(75):8-13.

16. Bolhari J, Ramezanzadeh F, Abedininia N, Naghizadeh MM, Pahlavani H, Saberi M. The survey of divorce incidence in divorce applicants in Tehran. J Family Reprod Health. 2012;6(3):129-137.

17. Sociological analysis of divorce in Iran. Tehran: Islamic Parliament Research Center; 2011. [Persian].

18. Islamic Parliament Research Center. Study the growth of birth, marriage and divorce in Iran (Challenges and Solutions). Tehran: Islamic Parliament Research Center; 2011. [Persian].

19. Mohammadi K, Heydari M, Faghihzadeh S. Validity of Persian Female Sexual Function Index (FSFI). Payesh. 2008;2(7):269-278. [Persian].

20. Bolourian Z, Ganjloo J. Evaluating sexual dysfunction and some related factors in women attending Sabzevar health care centers. J Reprod Infertil. 2007;8(2):163-170.

21. Basson R. Female sexual response: the role of drugs in the management of sexual dysfunction. Obstet Gynecol. 2001;98(2):350-353.

22. Scott VC, Sandberg JG, Harper JM, Miller RB. The Impact of Depressive Symptoms and Health on Sexual Satisfaction for Older Couples: Implications for Clinicians. Contemp Fam Ther. 2012;34(3):376-390. doi:10.1007/s10591-0129198-2
23. Elnashar AM, El-Dien Ibrahim M, El-Desoky MM, Ali OM, El-Sayd Mohamed Hassan M. Female sexual dysfunction in Lower Egypt. BJOG. 2007;114(2):201-206. doi:10.1111/ j.1471-0528.2006.01106.x

24. Mazinani R, Akbari Mehr M, Kaskian A, Kashanian M. Evaluation of Prevalence of sexual dysfunctions and its related factors in women. Razi Journal of Medical Sciences 2013; 19(105): 59-66.

25. Shokrollahi P, Mirmohamadi M, Mehrabi F, Babaei G. Prevalence of sexual dysfunction in women seeking services at family planning centers in Tehran. J Sex Marital Ther. 1999;25(3):211-215. doi:10.1080/00926239908403995

26. Yekeh Fallah L, Goodarzi M. Prevalence of sexual dysfunction and related factors among married couples in Qazvin. Journal of Qazvin University of Medical Sciences. 2009;13(1):49-55.

27. Hoseini Tabaghdehi MA, Haji Kazemi EA, Hoseini F. The Relative Frequency of Sexual Dysfunction and Some related Factors in the Women Referred to the Health Centers of Sari City (2006). Journal of Mazandaran University of Medical Sciences. 2012;22(91):102-107.

28. Sehhatie F, Malakouti G, Mirghafourvand M, Khalilpoor S. Sexual function and its relationship to general health in postpartum women. Journal of Womens Health, Issues and Care 2016; 5:2. doi:10.4172/2325-9795.1000221.

29. Pourakbaran E, Amin Yazdi SA. A study of sexual functioning and marital satisfaction in women with and without history of labor. Journal of Fundamentals of Mental Health. 2015;17(4):202-208.

30. Nourouzi M, Bashardoust N, Farmahini Farahani M. Postpartum physico sexual problems and related factors. Journal of Shahrekord Uuniversity of Medical Sciences. 2005;7(3):28-32

31. Karbasi-Afshar R, Saadat SH, Izadi M. Erectile Dysfunction and Cardiovascular Complications. Int $\mathrm{J}$ Med Rev. 2014;1(3):125-131

(C) 2018 The Author (s); This is an open-access article distributed under the terms of the Creative Commons Attribution License (http://creativecommons.org/licenses/by/4.0), which permits unrestricted use, distribution, and reproduction in any medium, provided the original work is properly cited. 